\title{
Clinical Practice Status of Sentinel Lymph Node Biopsy for Early-Stage Breast Cancer Patients in China: A Multicenter Study
}

This article was published in the following Dove Press journal:

Clinical Epidemiology

\author{
Juliang Zhang ${ }^{1 * *}$ \\ Ting Wang',* \\ Changjiao Yan' \\ Meiling Huang' \\ Zhimin Fan ${ }^{2}$ \\ Rui Ling (D) \\ 'Department of Thyroid, Breast and \\ Vascular Surgery, Xijing Hospital, The \\ Fourth Military Medical University, Xi'an, \\ Shaanxi 710032, People's Republic of \\ China; ${ }^{2}$ Department of Breast Surgery, \\ The First Affiliated Hospital of Jilin \\ University, Changchun, Jilin I3002I, \\ People's Republic of China
}

*These authors contributed equally to this work
Correspondence: Rui Ling; Zhimin Fan Email lingruiaoxue@I26.com; fanzhimn@I63.com
Background: Sentinel lymph node biopsy (SLNB) was introduced over 30 years ago, but the application of SLNB in China is unclear. This study aimed to explore the real-world implementation of SLNB among early-stage breast cancer patients in China.

Methods: A multi-center, retrospective study was conducted among primary breast cancer patients from 37 hospitals in China in 2018. Their clinical data were collected and analyzed, including the implementation status of SLNB in China, subsequent processing of sentinel lymph nodes (SLNs) containing metastases, and the effect of neoadjuvant chemotherapy (NAC) on SLNB.

Results: SLNB surgery was performed on $43.5 \%$ of early-stage breast cancer patients in China and 11,942 patients who underwent SLNB were enrolled in this study. The majority of SLNBs were performed using a single mapping agent. A combination of blue dye and radiotracer or fluorescence imaging was used in only $14.9 \%$ of patients. The mean (SD) number of resected SLNs was 4.0 (2.1). For the patients with 1 or 2 positive SLNs, 83.0\% of them continued to receive axillary lymph node dissection (ALND), while others did not. For the patients with three or more positive SLNs, 97.2\% of them continued to receive ALND, among which $82.9 \%$ accepted radiotherapy simultaneously. Of the patients who underwent SLN surgery, 5.5\% $(654 / 11,942)$ were receiving NAC. Among them, 51.9\% received SLNB before NAC, and the rest received SLNB after NAC. In biopsy-proven positive nodes, $64.7 \%$ positive SLNs turned negative after NAC.

Conclusion: SLNB has been promoted in China, but it is not widely used compared to in developed countries. Furthermore, the usage of the dual tracer technique in SLNB is not high. Chinese breast surgeons are more conservative regarding the omission of ALND in 1 or 2 SLNs-positive patients.

Keywords: sentinel lymph node, early-stage breast cancer, real word data

\section{Introduction}

In primary breast cancer, identifying the presence of axillary node metastasis is essential for breast cancer evaluation and staging establishment. ${ }^{1}$ Axillary staging remains the standard of care for all breast cancers, providing information important for individualized surgical treatment. Due to the limited sensitivity of imaging techniques in axillary lymph node detection, the axilla must be explored surgically. However, axillary lymph node dissection (ALND) may result in lymphedema, nerve injury, shoulder dysfunction, and other short-term and long-term complications limiting functionality and reducing quality of life. ${ }^{2}$ 
With the advent of sentinel lymph node biopsy (SLNB), surgical methods for accurately staging axilla in patients with early-stage breast cancer have become progressively less extensive. ${ }^{3}$ SLNB has supplanted ALND as the gold standard for axillary lymph node staging in clinically and radiologically node-negative breast cancer. ${ }^{4}$ SLNB staging can reduce surgical morbidity in terms of shoulder dysfunction and lymphedema, without affecting diagnostic accuracy and prognostic information. ${ }^{5}$

Recently, several SLNB methods have been reported with good efficacy and safety data, including methylene blue dye, nanocarbon, near-infrared (NIR) fluorescence imaging, 99 m-Technetium (99-Tc), and dual tracing modality. ${ }^{6,7}$ Mapping sentinel lymph node locations with methylene blue dye alone results in an acceptable identification rate but an excessive false negative rate, according to the American Society of Breast Surgeons' recommendations. ${ }^{8}$ NIR fluorescence imaging using indocyanine green (ICG) has been proven to have valid diagnostic performance for sentinel lymph node detection and showed a trend toward better axilla staging. ${ }^{9}$ Dual tracing modality using ICG plus methylene blue has been demonstrated to improve the mapping performance and exhibit great potential as an alternative to traditional standard mapping methods. ${ }^{10}$ Although mapping tracers are constantly evolving, their application in the real world is still unclear. Furthermore, the findings of the American College of Surgeons Oncology Group (ACOSOG) Z0011 randomized clinical trial do not support the routine use of ALND in patients with 1 or 2 metastases sentinel lymph nodes (SLNs). ${ }^{11}$ After the publication of Z0011, the proportion of 1-2 SLNs-positive patients who avoid ALND is still unknown in China. Due to the lower detection rate and a higher false-negative rate compared with SLNB done before neoadjuvant chemotherapy (NAC), ${ }^{12}$ the feasibility of performing SLNB after NAC is controversial. The proportion of patients who have SLNB performed after NAC is unclear in China.

To answer these questions, we conducted a multicenter, retrospective study from a prospectively maintained database. The real-world data of SLNB in China were investigated among women with early-stage breast cancer from January 2018 to December 2018. SLNB tracer method, number of resected SLNs, metastases rate of SLNs, number of metastases SLNs, subsequent processing of metastases SLNs, and effect of neoadjuvant chemotherapy on SLNB were analyzed. To our knowledge, this is the largest study on SLNB in early-stage breast cancer in China so far.

\section{Materials and Methods}

\section{Patients}

A total of 28,132 patients with breast cancer admitted to 37 medical centers from January 2018 to December 2018 were assessed for eligibility. Among them, 12,233 received SLNB surgery and finally, 11,942 were enrolled in this study. Inclusion criteria: (1) breast cancer confirmed by core needle biopsy, (2) SLNB with or without ALND, (3) receiving conventional systematic treatment or regional treatment, (4) complete medical record. Exclusion criteria: (1) IV stage breast cancer, (2) combined with a secondary invasive malignant tumor, (3) diagnosed with another serious disease, including congestive heart failure, unstable angina pectoris, myocardial infarction, high-risk uncontrollable heart rate disorder or other serious cardiovascular diseases within 6 months, (4) difficulty breathing at rest or need oxygen therapy, (5) severe infection, (6) uncontrolled diabetes, (7) serious psychological or mental disorders, (8) poor compliance. We retrospectively collected detailed SLNB and clinicopathologic data from patient records. All enrolled patients provided informed consent. Protocols for SLNB and evaluation of data in the retrospective study followed the principles of the Declaration of Helsinki and was approved by the Ethical Committee of Xijing Hospital, The Fourth Military Medical University (KY20192114-C-1).

\section{Sentinel Lymph Node Evaluation}

The SLNB technique was left to the discretion of the operating surgeon. It was performed using blue dye, carbon nanoparticles, indocyanine green, lymphoscintigraphy, or dual tracing modality (a combination of blue dye and radiotracer or indocyanine green). Hot, blue, black, fluorescent, or palpable nodes were removed. Then, surgical specimens were examined by pathologists through hematoxylin-eosin staining to determine whether sentinel lymph nodes were metastatic. Sentinel lymph nodes were considered to be metastatic or positive if they contained a macrometastasis (deposit $>2 \mathrm{~mm}$ ) and a micro-metastasis (deposit $\geq 0.2 \mathrm{~mm}$ to $2 \mathrm{~mm}$ ). The metastasis rate $=$ the number of patients who had metastasis sentinel lymph nodes/the number of patients who underwent SLNB $\times$ $100 \%$. Patients underwent preoperative clinical assessment of lymph node status by palpation, imaging examination (including ultrasound, mammography, or magnetic resonance imaging), or ultrasound-guided core-needle biopsy. The statuses of estrogen receptor (ER), progesterone 
receptor (PR), and human epidermal growth factor 2 (HER2) were determined using an immunohistochemical (IHC) assay. Positive ER and PR status was defined as $\geqq 1 \%$ of tumor cells presenting positive nuclear staining. The results of HER2 were scored semi-quantitatively according to the estimated percentage of positively stained tumor cell nuclei and the intensity of nuclear staining (for no staining, $1+$ for weak staining, $2+$ for moderate staining, and $3+$ for strong staining). A fluorescence in situ hybridization (FISH) test was recommended to "2 +" patients to determine the HER2 expression status. Results of "-" or "1+" were classified as HER2 negative and " $3+$ " or "amplification of HER2 confirmed by FISH" as positive. The molecular subtype is divided into the following four categories: Luminal A (ER+, PR+, HER2, ki-67 $\leq 20 \%$ ), Luminal B (ER+ and/or PR+, HER2-, ki$67>20 \%$; ER+ and/or PR+, HER2+), HER2 positive (ER-, PR-, HER2+), or triple-negative (ER-, PR-, HER2-). We judged patients to be node-negative (cN0) when palpation and imaging examination showed no suspicious nodes, or imaging examination showed suspicious nodes but the core-needle biopsy showed negative.

\section{Statistical Analysis}

All statistical analyses were performed with SPSS, version 22.0 (SPSS Inc., Chicago, IL, USA). Categorical data were summarized with frequencies and percentages. Continuous data were represented by mean (SD). For continuous variables, Mann-Whitney $U$-test was used to compare the medians with results expressed as $95 \%$ confidence intervals $(95 \% \mathrm{CI})$ of median difference. For categorical variables, percentages were compared using ratio statistics with results expressed as $95 \% \mathrm{CI}$.

\section{Results}

\section{Patient Populations and Characteristics}

A total of 11,942 breast cancer patients who underwent SLN surgery as part of their surgical treatment from 37 medical centers in China in 2018 were enrolled in this study. Table 1 shows the characteristic of these patients.

\section{Implementation Status of SLNB in China}

Among 28,132 breast cancer patients in the 37 medical centers, $43.5 \%(12,233 / 28,132)$ received SLNB surgery. As shown in Figure 1, the majority of SLNBs were performed using a single mapping agent, including blue dye, carbon nanoparticles, indocyanine green, or lymphoscintigraphy.
Table I Characteristics of the Patients and Tumors

\begin{tabular}{|l|l|}
\hline Characteristic & Number (n,\% or Mean (SD)) \\
\hline Number of patients & 11,942 \\
Age at diagnosis & $51.40(11.18)$ \\
\hline Operative procedure & \\
Lumpectomy & $3604(30.2 \%)$ \\
Mastectomy & $7595(63.6 \%)$ \\
Reconstruction & $581(4.8 \%)$ \\
Unknown & $162(1.4 \%)$ \\
\hline Histology & \\
Ductal carcinoma in situ (DCIS) & $1585(13.3 \%)$ \\
Lobular carcinoma in situ & $20(0.2 \%)$ \\
Invasive ductal carcinoma & $8718(73.0 \%)$ \\
Invasive lobular carcinoma & $354(3.0 \%)$ \\
Other & $1103(9.2 \%)$ \\
Unknown & $162(1.4 \%)$ \\
\hline Molecular subtype & \\
Luminal A & $3208(26.9 \%)$ \\
Luminal B & $4203(35.2 \%)$ \\
Her2-enriched & $1333(11.2 \%)$ \\
Triple-negative & $1138(9.5 \%)$ \\
Unknown & $2060(17.3 \%)$ \\
\hline Tumor size & \\
Tis & $1292(10.8 \%)$ \\
TI & $5790(48.5 \%)$ \\
T2 & $145(1.2 \%)$ \\
T3 & $23(0.2 \%)$ \\
T4 & $1479(12.4 \%)$ \\
Unknown & \\
\hline
\end{tabular}

A combination of blue dye and radiotracer or fluorescent dye was used only in $14.9 \%$ of SLNBs. The mean (SD) number of resected SLNs was 4.0 (2.1). According to the detected number $(\mathrm{N})$ of SLNs, the patients were divided into three groups of $\mathrm{N}=1-3,4-6$, and $\geq 7$. As shown in Table 2 , when a single mapping agent was used, the percentage of resected SLNs in 4 to $6(45.8 \%, 95 \% \mathrm{CI}=44.8-46.9 \%)$ was higher than when mapping was performed by a combination of blue dye and radiotracer $(35.1 \%, 95 \% \mathrm{CI}=32.7-37.5 \%)$. The percentage of resected SLNs more than 7, the metastases rate of SLNs and the number of metastases SLNs using single tracer or dual-tracer were similar.

\section{Metastases of SLNs and Subsequent Processing}

The incidence of SLNs metastases was 17.5\% $(2093 / 11,942)$ in patients who underwent SLNB. The mean (SD) number of metastatic SLNs was 1.6 (1.1). As shown in Table 3, 86.4\% 


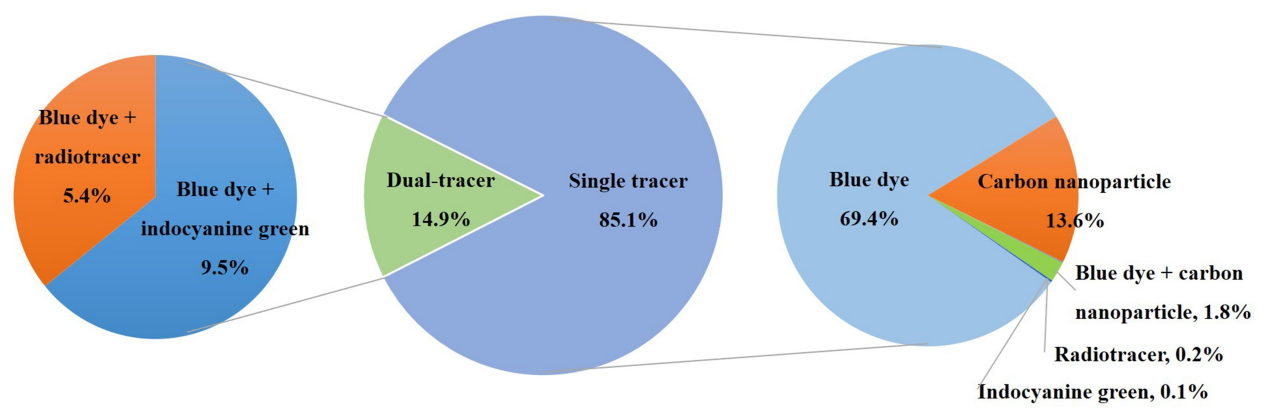

Figure I The tracer methods used in II,942 patients to perform SLNB. Dual-tracer refers to a combination of blue dye and radiotracer or fluorescent dye.

(95\% CI $=85.6-87.2 \%)$ of patients with cN0 had no metastatic SLNs, and $49.1 \%(95 \% \mathrm{CI}=45.9-52.4 \%)$ of patients with $\mathrm{cN} 1$ had no metastatic SLNs. Only 7.9\% (95\% CI = $6.1-9.6 \%$ ) of patients with $\mathrm{cN} 1$ had three or more metastatic SLNs. For the patients with 1-2 positive SLNs, $83.0 \%$ of them continued to receive ALND, among which $65.0 \%$ accepted radiotherapy simultaneously. While $13.7 \%$ of patients with 1-2 metastatic SLNs received radiotherapy only, $3.3 \%$ of patients were not treated after 1-2 metastatic SLNs were detected. For the patients with three or more metastatic SLNs, $97.2 \%$ of them continued to receive ALND, among which $82.9 \%$ accepted radiotherapy simultaneously. And $1.1 \%$ of patients with three or more metastatic SLNs only received radiotherapy.

\section{Effect of Neoadjuvant Chemotherapy on SLNB}

There were $654(5.5 \%)$ patients who received SLNB as well as neoadjuvant chemotherapy (NAC). Among them,
$51.9 \%$ of patients received SLNB before NAC, and the rest received SLNB after NAC. The mean (SD) number of resected SLNs was 4.0 (2.2) in the NAC group vs 3.9 (2.1) in the non-NAC group. As shown in Table 4, the metastases rate of SLNs before NAC $(28.8 \%, 95 \% \mathrm{CI}=23.0$ $34.6 \%)$ was higher than that after NAC $(14.2 \%, 95 \% \mathrm{CI}=$ 9.5-18.8\%). But performing SLNB before or after NAC did not affect number of resected SLNs (median 4.0 vs 4.0, median difference $=0.069,95 \% \mathrm{CI}$ of the difference $=$ 0.045-0.091) and metastases SLNs (median 1.0 vs 1.0 , median difference $=0.682,95 \% \mathrm{CI}$ of the difference $=$ $0.563-0.750$ ). For patients with biopsy-proven nodepositive breast cancer, $45.9 \%$ underwent SLNB surgery after NAC. A combination of blue dye and radiotracer or fluorescent dye was used in $48.6 \%$. After NAC, $64.7 \%$ positive SLNs turned negative.

\section{Discussion}

SLNB has established its role as the standard surgical procedure for staging clinically negative nodes in patients

Table 2 The Effects of Tracer Method on the Number of Resected SLNs, Metastases Rate of SLNs, and Number of Metastases SLNs

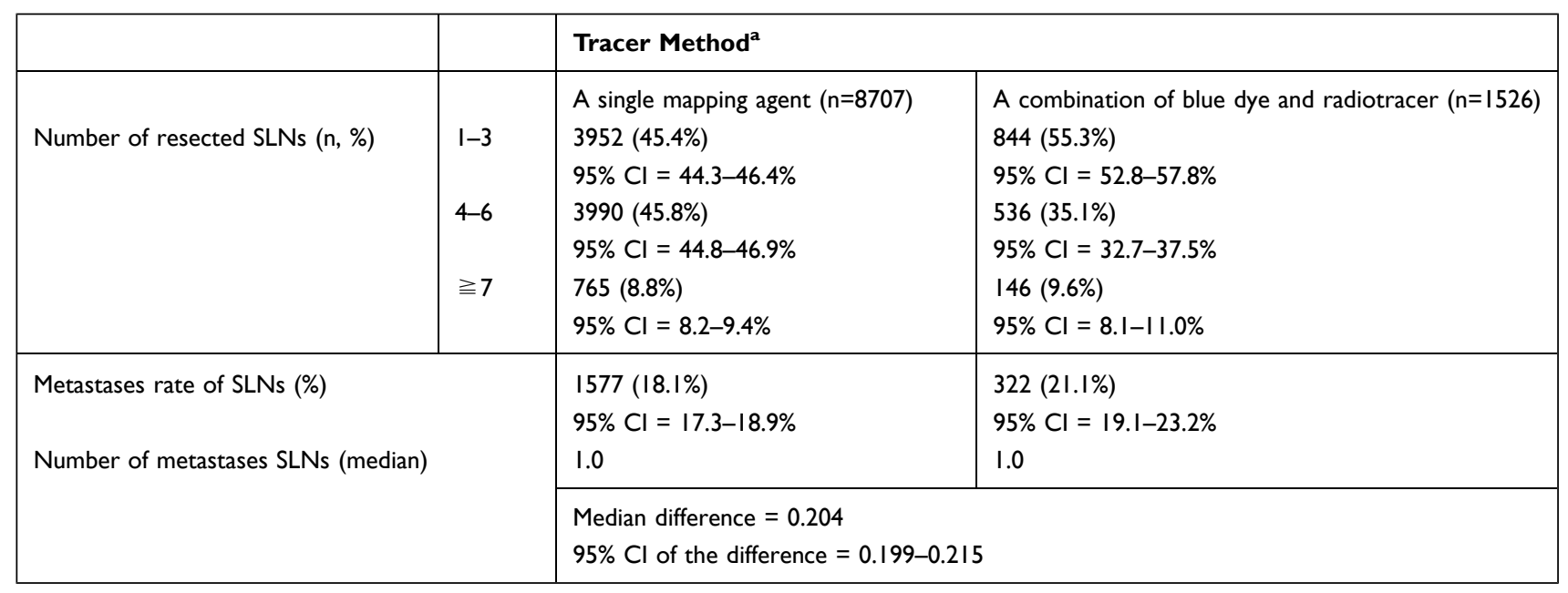

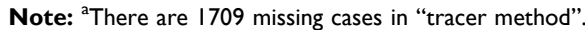

Abbreviation: $95 \% \mathrm{Cl}, 95 \%$ confidence interval. 
Table 3 Number of SLN Metastases Classified by Clinical Node States

\begin{tabular}{|l|l|l|l|}
\hline \multirow{2}{*}{$\begin{array}{l}\text { Clinical Node } \\
\text { States* }\end{array}$} & \multicolumn{3}{|l|}{ Number of SLN Metastases, N(\%) } \\
\cline { 2 - 4 } & $\mathbf{0}$ & $\mathrm{I}-2$ & $\geqq 3$ \\
\hline $\begin{array}{l}\text { Clinically node- } \\
\text { negative (cN0) }\end{array}$ & $6 \mathrm{II}(86.4 \%)$ & $\begin{array}{l}878(12.4 \%) \\
95 \%\end{array}$ & $\begin{array}{l}86(\mathrm{I} .2 \%) \\
95 \%\end{array}$ \\
& $\mathrm{Cl}=85.6-87.2 \%$ & $\mathrm{Cl}=1 \mathrm{I} .6-13.2 \%$ & $\mathrm{Cl}=1.0-1.5 \%$ \\
\hline $\begin{array}{l}\text { Clinically node- } \\
\text { positive (cNI } \\
\text { or cN2) }\end{array}$ & $448(49.1 \%)$ & $392(43.0 \%)$ & $72(7.9 \%)$ \\
\hline
\end{tabular}

Notes: *3294 patients lacked information of clinical node states. And this table does not include 654 patients who underwent neoadjuvant chemotherapy.

Table 4 The Number of Resected SLNs, The Metastasis Rate of SLNs, and the Number of Metastases SLNs According to Time of Neoadjuvant Chemotherapy (NAC)

\begin{tabular}{|c|c|c|c|}
\hline & $\begin{array}{l}\text { Number } \\
\text { of } \\
\text { Resected } \\
\text { SLNs } \\
\text { (Median) }\end{array}$ & $\begin{array}{l}\text { Metastases } \\
\text { Rate of SLNs } \\
\text { (\%) }\end{array}$ & $\begin{array}{l}\text { Number of } \\
\text { Metastases } \\
\text { SLNs } \\
\text { (Median) }\end{array}$ \\
\hline $\begin{array}{l}\text { Underwent SLNB } \\
\text { before NAC }\end{array}$ & 4.0 & $\begin{array}{l}28.8 \%(95 \% \\
\mathrm{Cl}=23.0-34.6 \%)\end{array}$ & 1.0 \\
\hline $\begin{array}{l}\text { Underwent SLNB } \\
\text { after NAC }\end{array}$ & 4.0 & $\begin{array}{l}14.2 \%(95 \% \mathrm{Cl}= \\
9.5-18.8 \%)\end{array}$ & 1.0 \\
\hline Median difference & 0.069 & I & 0.682 \\
\hline $\begin{array}{l}95 \% \text { confidence } \\
\text { interval of the } \\
\text { difference }\end{array}$ & $0.045-0.091$ & I & $0.563-0.750$ \\
\hline
\end{tabular}

with early-stage breast cancer. ${ }^{2}$ The safety, clinical value, and feasibility of the SLNB have been validated in a robust body of randomized controlled trials. ${ }^{13,14}$ However, more than 30 years after its introduction, ${ }^{15}$ the application of SLNB in the real world in China is unclear. In this study, we investigated the application of SLNB in early breast cancer patients from 37 medical centers in China who underwent SLN surgery as part of their surgical treatment. SLNB tracer method, metastases rate and number of SLNs, subsequent processing of metastases SLNs, and effect of neoadjuvant chemotherapy on SLNB in the real world were described in this study.

Among 28,132 breast cancer patients from 37 medical centers in China in 2018, 43.5\% received SLNB surgery. Previous studies showed that SLNB was more prevalent in developed countries: the use of SLNB increased from $11 \%$ to $59 \%$ from 1998 to 2004 in developed countries. ${ }^{16}$ The use of SLNB as definitive axillary staging increased from
$72 \%$ to $93 \%$ between 2011 and 2015 in Dutch nondistant metastatic breast cancer patients. ${ }^{17}$ From 2012 to 2013, upfront SLNB increased from $90.1 \%$ to $91.4 \%$ in clinical T1-2N0 breast cancer patients who underwent breastconserving surgery in the United States. ${ }^{18}$ By contrast, the prevalence of SLNB was less than 5\% in 2007 in China, and even lower in other developing countries. ${ }^{19}$ Our study suggested that the implementation of SLNB has increased, but it is not widely used compared to in developed countries. In terms of the tracer method, a combination of blue dye and radiotracer or indocyanine green was used only in $14.9 \%$ of SLNBs. For patients with biopsy-proven node-positive breast cancer and receiving NAC, the utilization rate of dual tracers increased to $48.6 \%$. The combination use of radioisotope and blue dye was recognized as the gold standard with the highest identification rate of up to $96 \%$ and lowest false-negative rate of $5 \% .{ }^{20}$ A combination of indocyanine green and blue dye has been proven to offer comparable detection sensitivity when compared to conventional mapping strategies (blue dye in conjunction with a radioisotope) in a prospective randomized study. ${ }^{21}$ And the dual technique is particularly recommended for SLNB after NAC. ${ }^{22}$ However, neither a combination of blue dye with radioisotope nor with indocyanine green was prevalent in China. And the magnetic technology used in clinical trials has not yet appeared in the real world. ${ }^{23}$ A single tracer especially blue dye is so far the first choice of SLNB in China, because it is safe, cheap and does not need the nuclear medicine department and gamma probes. In addition, concern about the hazards of radiation exposure is also an obstacle to the use of the combined method. Nevertheless, the mean (SD) number of resected SLNs reached 4.0 (2.1) in China, which is enough for treatment.

After the publication of ACOSOG-Z0011 results, the pattern of axillary surgery changed. ${ }^{24}$ Variations in patterns of axillary surgery are present in the post ACOSOGZ0011 era. Our results showed that $83 \%$ of early-stage breast cancer patients with 1-2 positive sentinel nodes underwent ALND in China in 2018. Robinson et al reported that the percentage of ALND decreased from $84 \%$ to $63 \%$ in breast cancer patients treated with SLNB after the publication of Z0011 results. ${ }^{25}$ In Japan, SLNB followed by ALND was performed in $73.9 \%$ of breast cancer cases in $2014 .{ }^{26}$ In the Netherlands, the use of completion ALND decreased from $75 \%$ to $17 \%$ in cT12N0 sentinel node-positive patients between 2011 and 2015. ${ }^{17}$ In the United States, $31.2 \%$ of breast cancer 
patients with 1 to 2 positive sentinel nodes received completion ALND in 2013. ${ }^{18}$ These results suggest that Chinese breast surgeons are more conservative regarding the omission of ALND in 1 or 2 positive SLNs.

The use of NAC has been extended to evaluate the effectiveness of systemic therapy early and to offer more conservative breast surgery. ${ }^{27}$ However, dissection of SLNs is more difficult after NAC owing to the change in lymphatic drainage patterns. ${ }^{28}$ Therefore, the accuracy and reliability of SLNB after NAC remain questionable. Factors that have been shown to decrease false-negative rates (FNR) include using dual mapping agents, resecting more SLNs, and placing clips in suspicious nodes at the time of biopsy. ${ }^{29}$ In a meta-analysis of SLNB after NAC in patients with initial biopsy-proven node positive breast cancer, the FNR was $20 \%$ with one node removed, $12 \%$ with two nodes removed, and $4 \%$ with three or more nodes removed. ${ }^{30}$ In our study, the median number of resected SLNs after NAC was 4.0 , and dual mapping agents were used for $48.6 \%$ of patients. But the data of whether the node was clipped are unavailable. In addition, the limitation of this study is the lack of data of postoperative lymph node dissection, so a false-negative rate cannot be obtained. In this study, for biopsy-proven node-positive breast cancer patients, $64.7 \%$ of positive SLNs turned negative after NAC. The results indicate that approximately two-thirds of node-positive patients could be exempted from ALND after NAC, but the safety of the omission of ALND after NAC remains unclear.

In conclusion, SLNB has been promoted in China in 2018, but it is not widely used compared to in developed countries. Furthermore, the usage of a dual tracer technique in SLNB is not high. And Chinese breast surgeons are more conservative regarding the omission of ALND in 1 or 2 positive SLNs. In addition, the proportion of patients who performed SLNB before and after NAC is similar. And approximately twothirds of positive nodes turn negative after NAC.

\section{Abbreviations}

SLNB, sentinel lymph node biopsy; SLNs, sentinel lymph nodes; ALND, axillary lymph node dissection; NAC, neoadjuvant chemotherapy; NIR, near-infrared; 99-Tc, 99 m-Technetium; ICG, indocyanine green.

\section{Data Sharing Statement}

Major findings from the study will be published in scientific manuscripts. The data will be made available on request. In order to preserve the privacy of the patients in compliance with local laws and regulations, data related to patient privacy (name, hospitalization number) will not be available.

\section{Ethics Approval and Informed Consent}

Protocols for SLNB and evaluation of data in the retrospective study followed the principles of the Declaration of Helsinki and was approved by the Ethical Committee of Xijing Hospital, The Fourth Military Medical University (KY20192114-C-1). The clinical trial ID of this study is NCT04156841.

\section{Acknowledgments}

The authors are grateful for the assistance of the Breast Surgery Group of the Chinese Medical Association Surgery Society and the data providers in Beijing Chaoyang Hospital, Peking University First Hospital, Peking University People's Hospital, Xuanwu Hospital of Capital Medical University, Beijing Friendship Hospital, The Second Hospital of Dalian Medical University, Fujian Medical University Union Hospital, The Obstetrics \& Gynecology Hospital of Fudan University, Huashan Hospital of Fudan University, Zhongshan Hospital of Fudan University, Gansu Provincial Hospital, Gansu Provincial Cancer Hospital, Affiliated Wudang Hospital of Guizhou Medical University, The Second Affiliated Hospital of Harbin Medical University, The Fourth Hospital of Hebei Medical University, Henan Cancer Hospital, The First Affiliated Hospital of Jilin University, Jilin Cancer Hospital, Jiangsu Province Hospital, The General Hospital of the People's Liberation Army, Xijing Hospital, The First Hospital of Lanzhou University, The Second Hospital of Lanzhou University, Southwest Hospital, The Second Affiliated Hospital of Nanchang University, Inner Mongolia Autonomous Region People's Hospital, Second Hospital of Shandong University, Shandong Provincial Western Hospital, Sichuan Provincial People's Hospital, Affiliated Cancer Hospital of Xinjiang Medical University, Yunnan Cancer Hospital, The First Affiliated Hospital of Zhejiang University, The Second Affiliated Hospital of Zhejiang University, The First Hospital of China Medical University, Shengjing Hospital of China Medical University, Xiangya Hospital Central South University, and Sun Yat-sen Memorial Hospital of Sun Yat-sen University. 


\section{Funding}

This paper was funded by Key R\&D Program of Shaanxi Province, China. (No. 2018ZDXM-SF-066).

\section{Disclosure}

The authors report no conflicts of interest in this work.

\section{References}

1. Ecanow JS, Abe H, Newstead GM, Ecanow DB, Jeske JM. Axillary staging of breast cancer: what the radiologist should know Radiographics. 2013;33(6):1589-1612. doi:10.1148/rg.336125060

2. Manca G, Rubello D, Tardelli E, et al. Sentinel lymph node biopsy in breast cancer: indications, contraindications, and controversies. Clin Nucl Med. 2016;41(2):126-133. doi:10.1097/RLU.0000000000000985

3. Jatoi I, Benson JR, Toi M. De-escalation of axillary surgery in early breast cancer. Lancet Oncol. 2016;17(10):e430-e441. doi:10.1016/ S1470-2045(16)30311-4

4. Charalampoudis P, Markopoulos C, Kovacs T. Controversies and recommendations regarding sentinel lymph node biopsy in primary breast cancer: a comprehensive review of current data. Eur J Surg Oncol. 2018;44(1):5-14. doi:10.1016/j.ejso.2017.10.215

5. Manca G, Tardelli E, Rubello D, et al. Sentinel lymph node biopsy in breast cancer: a technical and clinical appraisal. Nucl Med Commun. 2016;37(6):570-576. doi:10.1097/MNM.0000000000000489

6. Niebling MG, Pleijhuis RG, Bastiaannet E, Brouwers $A H$, van Dam GM, Hoekstra HJ. A systematic review and meta-analyses of sentinel lymph node identification in breast cancer and melanoma, a plea for tracer mapping. Eur J Surg Oncol. 2016;42(4):466-473. doi:10.1016/j.ejso.2015.12.007

7. Zengel B, Yararbas U, Sirinocak A, et al. Sentinel lymph node biopsy in breast cancer: review on various methodological approaches. Tumori. 2013;99(2):149-153. doi:10.1177/030089161309900205

8. Li J, Chen X, Qi M, Li Y, Metze K. Sentinel lymph node biopsy mapped with methylene blue dye alone in patients with breast cancer: a systematic review and meta-analysis. PLoS One. 2018;13(9): e0204364. doi:10.1371/journal.pone.0204364

9. Sugie T, Ikeda T, Kawaguchi A, Shimizu A, Toi M. Sentinel lymph node biopsy using indocyanine green fluorescence in early-stage breast cancer: a meta-analysis. Int J Clin Oncol. 2017;22(1):11-17. doi:10.1007/s10147-016-1064-z

10. Guo J, Yang H, Wang S, et al. Comparison of sentinel lymph node biopsy guided by indocyanine green, blue dye, and their combination in breast cancer patients: a prospective cohort study. World J Surg Oncol. 2017;15(1):196. doi:10.1186/s12957-017-1264-7

11. Giuliano AE, Ballman KV, McCall L, et al. Effect of axillary dissection vs no axillary dissection on 10-year overall survival among women with invasive breast cancer and sentinel node metastasis: the ACOSOG Z0011 (alliance) randomized clinical trial. JAMA. 2017;318(10):918-926. doi:10.1001/jama.2017.11470

12. Kuehn T, Bauerfeind I, Fehm T, et al. Sentinel-lymph-node biopsy in patients with breast cancer before and after neoadjuvant chemotherapy (SENTINA): a prospective, multicentre cohort study. Lancet Oncol. 2013;14(7):609-618. doi:10.1016/S1470-2045(13)70166-9

13. Canavese G, Bruzzi P, Catturich A, et al. Sentinel lymph node biopsy versus axillary dissection in node-negative early-stage breast cancer: 15-year follow-up update of a randomized clinical trial. Ann Surg Ancol. 2016;23(8):2494-2500.

14. Krag DN, Anderson SJ, Julian TB, et al. Sentinel-lymph-node resection compared with conventional axillary-lymph-node dissection in clinically node-negative patients with breast cancer: overall survival findings from the NSABP B-32 randomised Phase 3 trial. Lancet Oncol. 2010;11(10):927-933. doi:10.1016/S1470-2045(10)70207-2
15. Wiatrek R, Kruper L. Sentinel lymph node biopsy indications and controversies in breast cancer. Maturitas. 2011;69(1):7-10. doi:10.1016/j.maturitas.2011.02.006

16. Rescigno J, Zampell JC, Axelrod D. Patterns of axillary surgical care for breast cancer in the era of sentinel lymph node biopsy. Ann Surg Oncol. 2009;16(3):687-696. doi:10.1245/s10434-008-0195-5

17. Poodt IGM, Spronk PER, Vugts G, et al. Trends on axillary surgery in nondistant metastatic breast cancer patients treated between 2011 and 2015: a Dutch population-based study in the ACOSOG-Z0011 and AMAROS era. Ann Surg. 2018;268(6):1084-1090. doi:10.1097/ SLA.0000000000002440

18. Mann JM, Wu X, Christos P, Nagar H. The state of surgical axillary management and adjuvant radiotherapy for early-stage invasive breast cancer in the modern era. Clin Breast Cancer. 2018;18(4): e477-e493. doi:10.1016/j.clbc.2017.09.001

19. Leong SP, Shen ZZ, Liu TJ, et al. Is breast cancer the same disease in Asian and Western countries? World J Surg. 2010;34(10):2308-2324. doi:10.1007/s00268-010-0683-1

20. Cody HS. Clinical aspects of sentinel node biopsy. Breast Cancer Res. 2001;3(2):104-108. doi:10.1186/bcr280

21. Yuan L, Qi X, Zhang Y, et al. Comparison of sentinel lymph node detection performances using blue dye in conjunction with indocyanine green or radioisotope in breast cancer patients: a prospective single-center randomized study. Cancer Biol Med. 2018;15 (4):452-460. doi:10.20892/j.issn.2095-3941.2018.0270

22. Park S, Koo JS, Kim GM, et al. Feasibility of charcoal tattooing of cytology-proven metastatic axillary lymph node at diagnosis and sentinel lymph node biopsy after neoadjuvant chemotherapy in breast cancer patients. Cancer Res Treat. 2018;50(3):801-812. doi:10.4143/ crt.2017.210

23. Zada A, Peek MC, Ahmed M, et al. Meta-analysis of sentinel lymph node biopsy in breast cancer using the magnetic technique. Br J Surg. 2016;103(11):1409-1419. doi:10.1002/bjs.10283

24. Giuliano AE, Hunt KK, Ballman KV, et al. Axillary dissection vs no axillary dissection in women with invasive breast cancer and sentinel node metastasis: a randomized clinical trial. JAMA. 2011;305 (6):569-575. doi:10.1001/jama.2011.90

25. Robinson KA, Pockaj BA, Wasif N, Kaufman K, Gray RJ. Have the American College of Surgeons Oncology Group Z0011 trial results influenced the number of lymph nodes removed during sentinel lymph node dissection? Am J Surg. 2014;208(6):1060-1064; discussion 1063-1064. doi:10.1016/j.amjsurg.2014.08.009

26. Imoto S, Yamauchi C, Komoike Y, et al. Trends in axillary treatment for breast cancer patients undergoing sentinel lymph node biopsy as determined by a questionnaire from the Japanese Breast Cancer Society. Breast Cancer (Tokyo, Japan). 2017;24(3):427-432. doi:10.1007/s12282-016-0721-4

27. Duraes M, Guillot E, Seror J, Pouget N, Rouzier R. [Sentinel lymph node biopsy and neoadjuvant treatment in breast cancer]. Bull Cancer. 2017;104(10):892-901. doi:10.1016/j.bulcan.2017.08.003. French.

28. Fisher B, Brown A, Mamounas E, et al. Effect of preoperative chemotherapy on local-regional disease in women with operable breast cancer: findings from National Surgical Adjuvant Breast and Bowel Project B-18. J Clin Oncol. 1997;15(7):2483-2493. doi:10.1200/JCO.1997.15.7.2483

29. Boughey JC, Suman VJ, Mittendorf EA, et al. Factors affecting sentinel lymph node identification rate after neoadjuvant chemotherapy for breast cancer patients enrolled in ACOSOG Z1071 (Alliance). Ann Surg. 2015;261(3):547-552. doi:10.1097/ SLA.0000000000000551

30. Tee SR, Devane LA, Evoy D, et al. Meta-analysis of sentinel lymph node biopsy after neoadjuvant chemotherapy in patients with initial biopsy-proven node-positive breast cancer. Br J Surg. 2018;105 (12):1541-1552. doi:10.1002/bjs. 10986 


\section{Publish your work in this journal}

Clinical Epidemiology is an international, peer-reviewed, open access, online journal focusing on disease and drug epidemiology, identification of risk factors and screening procedures to develop optimal preventative initiatives and programs. Specific topics include: diagnosis, prognosis, treatment, screening, prevention, risk factor modification,

Submit your manuscript here: https://www.dovepress.com/clinical-epidemiology-journal systematic reviews, risk \& safety of medical interventions, epidemiology \& biostatistical methods, and evaluation of guidelines, translational medicine, health policies \& economic evaluations. The manuscript management system is completely online and includes a very quick and fair peer-review system, which is all easy to use. 